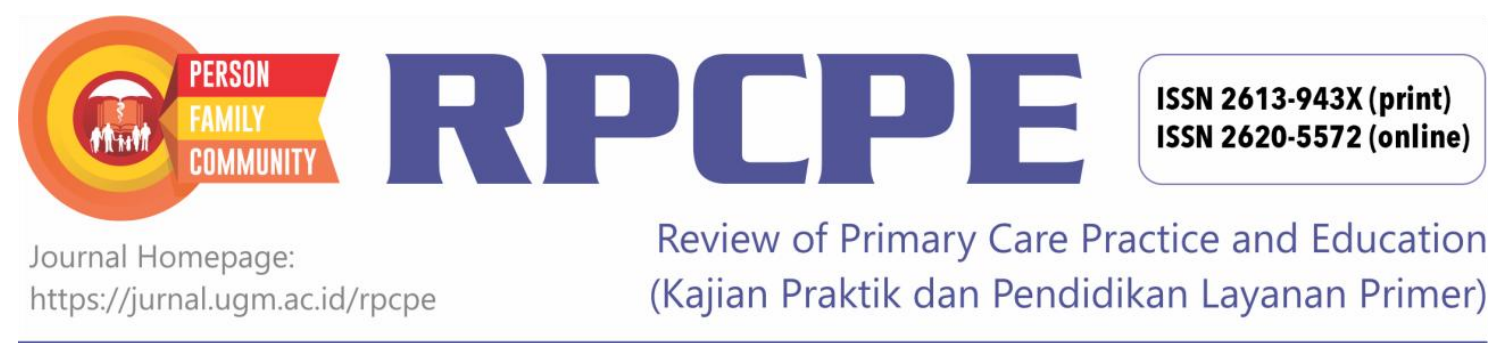

\title{
The Practice of Home-based Direct Observer Treatment of Patients with Positive Pulmonary Tuberculosis (DOT - TB) at Kebumen District of Indonesia
}

\author{
Timbul Pranoto ${ }^{1}$, Mora Claramita ${ }^{2}$, I Dewa Putu Pramantara ${ }^{3}$
}

\author{
${ }^{1}$ Puskesmas Kutowinangun (Community and Primary Health Care Center); Kebumen; Indonesia \\ ${ }^{2}$ Department of Family and Community Medicine; Faculty of Medicine, Public Health and Nursing; Universitas \\ Gadjah Mada; Indonesia \\ ${ }^{3}$ Department of Internal Medicine - Geriatric Division; Dr. Sardjito Hospital; Yogyakarta; Indonesia \\ Corresponding Author: \\ Timbul Pranoto: Puskesmas Kutowinangun, Jl. Yahya No.1 Kutowinangun, Kebumen, Jawa Tengah - 54393, Indonesia \\ Email: timbul.kbm@gmail.com
}

To cite this article:

Pranoto T, Claramita M, Pramantara IDP. The practice of home-based direct observer treatment of patients with positive pulmonary tuberculosis (DOT - TB) at Kebumen district of Indonesia. Rev Prim Care Prac and Educ. 2018; 1(2): 56-61.

\begin{abstract}
Background: Tuberculosis (TB) is a major global health problem, even though TB can be prevented and treated. The WHO Global Tuberculosis Report 2015 reported 1.5 million deaths from TB, of which 1.1 million were TB with HIV-negative and 0.4 million were HIV-positive tuberculosis. As many as $25 \%$ of deaths from TB were in productive ages between 15 to 54 years. Low adherence is a major cause of treatment failure, drop-out and the rising number of cases of MDR (Multi Drugs Resistance). Adherence is also a key to improve the cure rate of treatment of TB patients. Home-based Direct Observer Treatment of patients with positive pulmonary Tuberculosis (DOT-TB) or in Bahasa Indonesia also known as Pengawas Minum Obat (PMO) may improve adherence and increase the TB cure rate and success rate. The DOT-TB has tasks to oversee, remind, motivate and assist TB patients who are undergoing the treatment process. Background of DOT-TB in improving adherence needs to be explored by examining their experiences, which can be expressed by using a phenomenological qualitative study to explore the meaning and significance of their experiences. Objectives: This study aimed to explore the efforts of DOT-TB in performing their duties, the constraints arising from these efforts, benefits, and expectations of the DOT-TB position. Methods: This study applied a qualitative approach with descriptive phenomenological methodology. The number of informants were 21 people divided into groups of DOT-TB whose patients were perfectly recovered (7 people), groups of DOT-TB whose patients were drop-out or failed (7 people) and a group of 7 TB programmers. Each group participated in the Focus Group Discussion (FGD) for 90-120 minutes. Results: The results of the study indicate that the presence of DOT-TB is very important and necessary in the management of TB. Thebiggest challenge of DOT-TBs in performing their duties are communication barriers due to differences in hierarchy and social statusin the society. Their sense of hesitancy was a major challenge of DOT-TB in charge. According to respondents, the ideal DOT-TB is someone close to the patients, has patience, compassion, enough knowledge about TB and good communication skills. Conclusions: In order to perform their duties well, DOT-TBs require training concerning tuberculosis and communication.
\end{abstract}

Keywords: Home-based Direct Observer Treatment of Patients with Positive Pulmonary Tuberculosis (DOT - TB), Phenomenology, Tuberculosis

\section{BACKGROUND}

Tuberculosis (TB) is a major health problem globally, although $\mathrm{TB}$ is a preventable and treatable disease. The WHO TB - A Global Emergency declared TB as a global emergency since 1993. TB is one of the oldest diseases known to humans, and one of the major causes of death from infection in the world until today ${ }^{1}$. The incidence and mortality due to TB has been declining, but the disease remains the second leading cause of death from infectious disease globally after HIV/AIDS ${ }^{2}$.

The WHO Global Tuberculosis Report 2015 stated that 
in 2014 that there were 9.6 million new TB cases, with 5.4 million men, 3.2 million women and 1 million cases in children. According to the WHO, 153 countries have reported the Drug Resistant TB at the end of $2014^{3}$. The new cases of MDR TB were estimated to be 480,000 cases and deaths from MDR TB cases were estimated at 190,000. In 2014 there were 1.5 million deaths from TB, of which 1.1 million were HIV-negative TB and 0.4 million cases of HIV-positive TB. This number included 890,000 cases in men and 480,000 in women. As many as $25 \%$ of TB deaths are found in the productive ages between 15 to 54 years. Indonesia is one of 22 countries with a high burden of TB. The WHO reports more than $85 \%$ of new TB cases have been detected, of which Asia has 55\% and Africa $30 \%$ of cases. Pulmonary TB disease accounts for $25 \%$ of all deaths that can be prevented ${ }^{3}$. Southeast Asia carries the heaviest of the global TB burden which is as much as $38 \%$ of global TB cases. India (23\%), Indonesia (10\%) and China (10\%) are the largest contributor countries to global TB cases ${ }^{3}$. Adherence in TB management becomes crucial in $\mathrm{TB}$ recovery and the key to improve the treatment cure rate of TB patients ${ }^{4}$. Lack of adherence is a major cause of treatment failure, drop-out and increased cases of MDR ${ }^{5}$. Poor adherence with medication may increase the risk of morbidity, mortality and drug resistance occurrence ${ }^{6}$.

Home-based Direct Observer Treatment for TB patients (DOT-TB) is one factor that can improve adherence and healing of the TB patients, but not all DOT-TBs are knowledgeable and do their job well ${ }^{7}$. The DOT-TB tasks are monitoring, reminding, and motivating TB patients to take medication regularly until the completion of treatment. DOT-TBs' background in supporting pulmonary TB patients to improve adherence needs to be assessed by exploring the DOT-TB experience. Bogdan and Taylor in Moleong ${ }^{8}$ explained that qualitative methodology is a research procedure that produces descriptive data in the form of written or spoken words from people and observed behaviors. The DOT-TB experiences can be expressed by using the qualitative phenomenological study that has the ability to explore the meaning and significance of them ${ }^{9}$. This phenomenological method can describe symptoms or phenomena that appear as they are as the objective of research ${ }^{8}$. This method can be used with the aim of providing an opportunity for the DOT-TB to express their experience naturally in assisting patients with smear positive pulmonary TB. Therefore, the researchers are interested in conducting research on the experience of DOT-TB while accompanying pulmonary TB patients. The researchers aimed to explore more deeply the efforts that have been made by DOT-TBs in performing their duties, barriers of DOT-TBs in performing the task, the benefits, and the expectations of the position of the DOTTB in the Direct Observe Treatment Short (DOTS) course.

\section{RESEARCH METHODS}

This study is a qualitative study with a phenomenological approach and took place in Kebumen District Health Office at mid Java island. The group consisted of four community and primary health care centers (Puskesmas), one hospital and one Lung Disease Treatment Unit (Unit
Pengobatan Penyakit Paru/UP3). The informants in this study were the Direct Observer of Treatment (DOT) whose patients were positive pulmonary tuberculosis (TB) adult patients enrolled in the registered TB program of Kebumen District Health Office (DHO) from January 1st, 2014 until December $31^{\text {st }}$, 2014. The other participants were the health workers who work in the institutions where the patients were treated. The total of our participants was 21, consisting of 7 DOT-TBs whose patients were smear positive pulmonary TB and totally recovered, 7 DOT-TBs whose patients were smear positive pulmonary (Acid Fast Bacilli/AFB) TB and dropped-out or failed treatment and 7 pulmonary TB programmers.

The sampling technique used was purposive sampling. The criteria for our DOT-TBs were as follows: 1) age 1565 years; 2) minimum of elementary education; 3 ) signed informed consent; and 4) supervised the smear positive pulmonary TB patients between January-December 2014. For programmers of pulmonary TB the following criteria were used: 1) age between 25-58 years of age; 2 ) at least become TB programmers for 3 years; 3 ) minimum education Health Program in Vocational School; and 4) were willing and had signed an informed consent. The research was conducted at four selected Puskesmas, one hospital and one Lung Disease Treatment Unit in Kebumen District. The determination of the Puskesmas was based on the number of patients and represented the area. Research instruments used were interviews and Focus Group Discussion (FGD) guidelines. The FGDs divided according to groups lasted 90-120 minutes and were recorded with a voice recorder. The data were collected by researchers assisted by two psychologists who had previously been explained about the research objectives. The result of the research was edited and the coding then analyzed by analytical descriptive methods. Activities in the analysis included data reduction, data presentation and conclusion drawing / verification. The transcript results were read and analyzed by 4 coders, then grouped into themes and subthemes. The coders were two psychologists, 1 master of Clinical Study Program, and one lecturer who has experience in coding. The coding was discussed until it reached saturation of data. To reduce the bias, then the triangulation process was done, i.e. triangulation of sources and researchers.

\section{RESULTS}

From the 21 respondents, 20 respondents could attend the FGDs and 1 respondent could not attend. The respondent who could not participate is one of the Lung TB programmers from a local hospital. To obtain the complete data of the respondents, the researcher collected the data by giving the same question with the FGD in writing.

\section{DOT-TB's duties}

The opinions most widely expressed by respondents in this study were: a DOT-TB oversees, reminds and motivates TB patients to take the medication regularly. DOT-TBs are also in charge of delivering TB patients to treatment facilities. Some quotations from the participants' opinions are as follows: 
"So DOT - TB's duty is not only overseeing the medicine has been taken or not, but We have to motivate how to get medical treatment regularly, how to drink it, so We were not only asking 'Have You taken the medicine yet?', But if We gave the motivation, the patient felt as if they were cared for, so they become enthusiastic."

"My father already had His own consciousness to take medication. If He wants to be accompanied to the clinic, We will accompany Him",

\section{DOT-TB's barriers in performing the tasks}

Most respondents expressed having communication barriers with TB patients because of differences in social status and hierarchy in the family / society. They may have different perceptions that the nature of TB patients is difficult to accept information so that the message they would deliver may not easily be accepted and understood by the patients. This phenomenon caused a frustration feeling. Another obstacle was the DOT-TB did not have the time to actually perfom his / her duty and there was a lack of DOT-TB training. Some quotations from the participants' opinions are as follows:

"Yes, it's not comfortable. I got to say something like this, 'Grandma, don't You want to see your children and grandchildren longer? Why don't You take the medicine.' However, She is actually living alone, her family are in Yogyakarta...."

"Yes, I am confused what to do about it. It's a 'must' so I feel I hesitated"

"Actually (the DOT-TB duty) is very important, but because I am a man, a husband, I have a responsibility to make a living for my wife, I cannot watch her every day, so I just trust my wife, "if you want to recover, you must obey the rules'.",

"Yes, because the perception of people affected by TB tend to be from low-income household"

"I once suggested, but it is back again depending on the conditions, 'Madam, it must be funded, where will the funds come from?', 'We take from the BOK (Health Operational Support) whether this is possible or not, We can see again later. For the event, if We can, We will join it later, but if not We are going to looking for other funds'. But so far it has not been running, because there are such obstacles".

\section{Efforts to overcome obstacles}

The most common opinion submitted by the respondents is that the DOT-TBs overcome barriers by providing motivation, conducting home visits, and requesting assistance from people who are more respected by the TB patients.

Some quotations from the participants' opinions are as follows:

"From me uhmm, the problem is nobody can be relied upon at home, so I just entrust her. 'Anyway, if you want to recover, you must keep the spirit to be healthy, medications should be taken regularly'. Maybe because we don't have uhmm, in this case, there are only my wife and a child, the kid is in school."

'I remember, every afternoon 'oh I have to go there', to where the patient lives, I keep remembering, when it's 5 o'clock, I went straight there, 'Have You already prepared the medicine?', 'yes, it is ready'.,

\section{DOT-TB's benefits}

All respondents said that the pulmonary TB patients are in need of DOT-TB while undergoing the treatment process. Some quotes from the participants' opinions are as follows:

"Yes, we desperately need it." (almost all participants responded by justifying)"

"Well, I think DOT-TB should be there, because it is very important for the patient. If not, for example, the neighbors do not know if there are DOT-TB, they will not be considered. If there is a DOT-TB, it is definitely on the right track."

\section{DOT-TB's Type}

Respondents expressed their opinion that the expected DOT-TB is someone who has patience and tolerance for TB patients, has a sense of affection and passion in monitoring pulmonary tuberculosis patients. Another opinion was the DOT-TBs should come from the closest contacts of the TB patients, have understanding of TB knowledge, and communication capabilities. Some quotations from the participants' opinions are as follows:

"Patience was already certain, the second is compassion, an affection of DOT-TB. So, if we have patience, continuing our capacity as much as possible, given the affection of all kinds of patients but it's still hard, what can we do. There is still a limit for this, but we must really care for them, not just an affection verbally. Patience, compassion, all."

"The nearest persons, who are psychologically and respected by the patient"

"Maybe the ideal one is, first the person who has understanding of TB knowledge, second the knowledge about OAT (anti TB medicine). Sometimes patients also confused about OAT, side effects from it, because, OAT may attack liver function."

\section{DISCUSSIONS}

The results in this study showed that the presence of DOTTB is very important and necessary in TB management program with the DOTS strategy. The duration of treatment, the large number of medicine intake and the side effects can potentially decrease TB patients' obedience in undergoing the treatment process. This condition will facilitate the occurrence of dropout or failure of treatment. The existence of the DOT-TB will increase adherence of TB patient so that will also increase the recovery rate. DOT-TB can be one of the keys to success of TB treatment with DOTS strategy. DOT-TB can increase the rate of recovery rate and success rate in the treatment of TB patients with smear positive pulmonary $\mathrm{TB}^{10}$. Patients with DOT-TB have a greater recovery rate $(84 \%)$ than without 
DOT-TB $(80.1 \%)^{11}$. The existence of the DOT-TB will provide a level of treatment success that is significantly better $^{12,13}$. DOT-TB will improve TB patients' adherence to treatment ${ }^{14}$. The presence of DOT-TB improves healing rate by $72 \%$ and reduces the effects of loss of follow-up ${ }^{15}$.

The biggest obstacle experienced by DOT-TB is communication barriers due to differences in hierarchy and social status in society. Their sense of hesitancy or in Javanese known as 'ewuh pekewuh' becomes one of the obstacles for the DOT-TB in charge. Eastern cultures still embrace their stratum of hierarchy, both in terms of age, status, education, and economics in building a relationship. This could become an obstacle for good communication between the DOT-TB with tuberculosis patients. It takes the ability to build equity so that communication may work well. In Javanese culture "Yes" does not necessarily mean approval or indicate that patients already understand what is conveyed. It is often just an expression of modesty toward people from higher social stratum. Javanese culture is very influential in the DOT-TB communications with TB patients, for their hierarchical strata is considered higher so that patients often say "yes", just to be polite to someone higher up.

In Eastern cultures, non-verbal language plays an important role in the consultation process ${ }^{16}$. TB patients expect to receive good treatment, attention and want to be heard from beginning till end, especially when experiencing the side effects of medicine. This matter emphasizes that the DOT-TB should be able to take the active role to establish equality so that communication can go well. To overcome this problem, DOT-TBs need to acquire communication skills training in order to face obstacles due to cultural differences.

Another obstacle is the DOT-TBs do not have enough time to oversee TB patients because they have other responsibilities. Prolonged treatment, the amount of medicine and side effects of medications can decrease TB patients' adherence to treatment process. As a result, continuous supervision is needed by the DOT-TB. TB patients want a DOT-TB to be someone who has time to spare so they can keep an eye on the patient to take the medication regularly ${ }^{17}$. Strict supervision in the intensive treatment phase is essential to prevent drug resistance ${ }^{17}$.

The existence of the DOT-TB will improve TB patients' adherence to undergo every part of the treatment process ${ }^{14}$. Therefore, TB patients require an ideal DOT-TB. According to respondents, an ideal DOT-TB is someone who is close to TB patients in terms of both social distance and relationships. It takes patience of DOT-TBs and compassion for TB patients. Proximity is expected to bring patience and compassion to DOT-TBs with pulmonary TB patients so they can accompany and supervise TB patients for a long term in order to complete the process according to the treatment standard. The DOT-TB position is voluntary and unpaid work that requires patience in order to control TB in the long term ${ }^{17}$.

The DOT-TBs suggested that the ideal supervisor of TB monitoring is their family. Family relationships can meet the criteria of the close relationship with the patients. DOT-TB of the family members could also mean people staying at home or residing near the homes of people with TB. This allows the DOT-TB to monitor and remind TB patients to take medicine regularly every day. An ideal DOT-TB is the people who live close to the homes of patients, and willing to help voluntarily ${ }^{18}$. Usually the DOT-TB is the wife, husband, children, mother or father of TB patients ${ }^{19}$.

DOT-TBs who live at the same house are expected to have a deep emotional connection that can be more tolerant to assist and provide motivation to people with TB to continue the treatment. DOT-TBs for pulmonary TB at home provide a better level of success in DOTS programs. This success rate depends on their easy communication, culture and the same language and emotional support that is greatly needed by pulmonary TB patients ${ }^{20}$. DOT-TBs who come from family members have several advantages, among others being close to the patient so that at any moment can monitor taking medication, have an emotional attachment that patients feel more attention from their family, who are closer and more trusted, and give more attention to the patient ${ }^{21}$.

In addition, the DOT-TB is expected to be a person who has enough knowledge about $\mathrm{TB}$ and has good communication skills. The ideal DOT-TB understands the management of TB. A DOT-TB should have knowledge about pulmonary $\mathrm{TB}$, mode of transmission, prevention, duration of treatment and the side effects of drugs. When DOT-TB knowledge is good, it will affect the practice of the DOT-TBs in performing their duties. A good knowledge will positively affect the DOT-TB in providing education, motivation and counseling of TB patients, families and communities. Some behaviors will be influenced by among other predisposing factors such as their knowledge ${ }^{22}$. A person's good knowledge of an activity will cause them to have a positive attitude that is reflected in their liveliness when they follow the activity level of knowledge ${ }^{23}$. Level of knowledge positively has been significantly associated with DOT-TB practices in monitoring pulmonary TB patients ${ }^{19}$.

The ideal DOT-TB needs assistance/mentoring and requires training. DOT-TBs need to understand more about TB disease, how to diagnosis, treatment processes, drug side effects and complications of TB disease. In addition, DOT-TBs also need to be given the ability of communication techniques on how to carry out education and counseling. DOT-TBs need to be trained in effective communication techniques in order to establish equality with TB patients so that the health messages can be received well. The results showed that there had been no training and coaching for the DOT-TBs from the clinic or hospital. In addition, there has been no specific program of support and funding for mentoring DOT-TBs. During this time the DOT-TB gain knowledge about TB from health authorities of the TB patient to deliver treatment in the health facilities ${ }^{19}$. This shows the importance of training for family members of DOT-TB on how to provide 
support for people in DOTS programs ${ }^{15}$.

From this study, it can be recommended that the TB patients should be accompanied by a DOT-TB. A specific program training on TB and communication skills for DOT-TBs is highly necessary. The local District Health Offices should provide financial support for the development and training of the DOT-TBs, by regularly supporting the annual planning of the DHO. The next study will focus on the effective training of DOT-TBs, especially the training of communication in education and counseling techniques. Limitations of this study include the subjectivity of the researchers, the lack of understanding from moderators about TB disease and how the FGDs could only be done once due to time and cost constraints so the result was that key information from respondents may not have been conveyed. The researcher tried to overcome these limitations by extending the discussion time and asking the moderators to provide repeated questions to confirm the respondent's answer.

\section{CONCLUSIONS}

From this study, the researchers concluded that the position of the DOT-TB is very important and necessary in containment procedures for the TB disease with the DOTS strategy. DOT-TB tasks include supervising, reminding, delivering, educating, motivating and providing socialization to patients with TB. The biggest hurdle DOT-TBs experience is communication barriers due to differences in social status and hierarchical differences as well as the natural difficulties that TB patients have in receiving input. DOT-TBs need specific skills to overcome these barriers and the ideal is someone who has the patient nature, is affectionate, have enough time, are significant others, people who understand about TB and have good communication skills. To realize the ideal DOT-TB requires training concerning $\mathrm{TB}$ and communication skills for the DOT-TBs to be able to perform their tasks successfully.

\section{Acknowledgements}

We want to extend our thanks to the Director of Dr. Soedirman Kebumen Hospital, Head of UP3 Kebumen, Head of Puskesmas Ambal II, Head of Puskesmas Kutowinagun, Head of Puskesmas Kebumen III and Head of Puskesmas Karanganyar.

\section{Ethical Approval and Informed Consent}

This study was approved by the Medical and Health Research Ethical Committee (MHREC) from the Faculty of Medicine, Public Health and Nursing, Universitas Gadjah Mada, Yogyakarta with reference number KE/ FK/1290/EC/2016.

\section{Funding}

Self-funding study

\section{Availability of Data and Materials}

Please contact the author for the availability of data and material through the author's correspondence.

\section{Conflict of Interest}

There is no conflict of interest at the time of the study nor in the writing of this publication.

\section{REFERENCES}

1. Ritz N, Curtis N. Novel concepts in the epidemiology, diagnosis and prevention of childhood tuberculosis. Swiss Med Wkly. 2014 Sep 10;144(September):w14000.

2. Murray CJ, Ortblad KF, Guinovart C, Lim SS, Wolock TM, Roberts DA, Dansereau EA, Graetz N, Barber RM, Brown JC, Wang H. Global, regional, and national incidence and mortality for HIV, tuberculosis, and malaria during 1990-2013: a systematic analysis for the Global Burden of Disease Study 2013. The Lancet. 2014 Sep 13;384(9947):1005-70.

3. World Health Organization. Tuberculosis (TB) global tuberculosis report 2015. Geneva, Switzerland: World Health Organization. 2015.

4. Atif M, Sulaiman SA, Shafi AA, Muttalif AR, Ali I, Saleem F. Applying patient centered approach in management of pulmonary tuberculosis: a case report from Malaysia. Journal of Basic and Clinical Pharmacy. 2011 Jun;2(3):129-130.

5. Mkopi A, Range N, Lwilla F, Egwaga S, Schulze A, Geubbels E, van Leth F. Adherence to tuberculosis therapy among patients receiving home-based directly observed treatment: evidence from the United Republic of Tanzania. PloS One. 2012 Dec 19;7(12):e51828.

6. Jain A, Dixit P. Multidrug resistant to extensively drug resistant tuberculosis: what is next?. Journal of Biosciences. 2008 Nov 1;33(4):605-16

7. Department of Health Republic of Indonesia. National Guidelines for Medical Services, Tuberculosis Management. Jakarta. Ministry of Health Republic of Indonesia. 2013: 6-7.

8. Moleong LJ. Qualitative Research Methodology (Revised edition). Bandung: PT Remaja Rosdakarya. 2015.

9. Collins D, Hafidz F, Suraratdecha C. The economic burden of tuberculosisi in Indonesia. TB Care I - Management Science for Health. Cambridge: Management Sciences for Health. 2013: 31.

10. Egwaga S, Mkopi A, Range N, Haag-Arbenz V, Baraka A, Grewal P, Cobelens F, Mshinda H, Lwilla F, van Leth F. Patient-centred tuberculosis treatment delivery under programmatic conditions in Tanzania: a cohort study. BMC Medicine. 2009 Dec;7(1):80.

11. Ai X, Men K, Guo L, Zhang T, Zhao Y, Sun X, Zhang H, He G, van der Werf MJ, Van Den Hof S. Factors associated with low cure rate of tuberculosis in remote poor areas of Shaanxi Province, China: a case control study. BMC Public Health. 2010 Dec;10(1):112.

12. Soomro MH, Qadeer E, Khan MA, Morkve O. Treatment supporters and their impact on treatment outcomes in routine tuberculosis program conditions in Rawalpindi District, Pakistan. National Research Institute of Tuberculosis and Lung Disease, Iran. Tanaffos. 2012;11(3):15-22.

13. Nurhayati J. The relation of treatment adherence supporter to successful TB medication by DOTS at Dr. Kariadi Hospital Semarang. Medica Hospitalia-Journal of Clinical Medicine. 2014 Sep 30;2(1):54-7.

14. Muniroh N, Aisah S, Mifbakhuddin. Factors associated with tuberculosis disease (pulmonary tbc) in work area of Puskesmas Mangkang West Semarang. Journal of Community Nursing. 2013;1(1): 33-42.

15. Mlilo N, Sandy C, Harries AD, Kumar AM, Masuka N, NyathiB, Edginton M, Isaakidis P, Manzi M, Siziba N. Does the type of treatment supporter influence tuberculosis treatment outcomes in Zimbabwe?. Public Health Action. 2013 Jun 21;3(2):146-8.

16. Claramita M, Nugraheni MD, van Dalen J, van der Vleuten C. Doctor-patient communication in Southeast Asia: a different culture?. Adv Health Sci Educ. 2013 Mar 1;18(1):15-31.

17. Purwanta P. Characteristics of treatment observer desired by pulmonary tuberculosis patients in urban and rural Yogyakarta. Journal of Health Services Management. 2005;8(03):141-7.

18. Ministry of Health Republic of Indonesia. National Guidelines for Medical Services: Tuberculosis Management. Jakarta: Ministry of Health Republic of Indonesia. 2013.

19. Widjanarko B, Prabamurti PN, Widyaningsih N. Analysis of factors affecting medicine supervisory practices (DOT - TB) in the control of pulmonary tuberculosis patients in Semarang city. Journal of Indonesia Health Promotion. 2006;1(1):15-24.

20. Amril Y, Surjanto E, Suriadi, Baktiar A. Directly observed therapy (DOT) in the treatment of new pulmonary tuberculosis cases in BP4 
Surakarta. J Respir Indo. 2003;23(2):67-75.

21. Kusbiyantoro. Comparison of the Effectiveness of Health Cadres and Community Struggle as Drug Supervisor of Drugs on Drug Compliance and Sputum Conversion of Pulmonary Tuberculosis in Kebumen District [Thesis]. Yogyakarta: Postgraduate UGM. 2002.

22. Green LW, Kreuter MW, Deeds SG, Partridge KB, Bartlett E. Health Education Planning: A Diagnostic Approach. Tafal, Mamdy, \& Krisna (Translation). Jakarta: Development Project FKM UI. 2000.

23. Notoatmodjo S. Education and Health Behavior. Jakarta: Rineka Cipta. 2003. 